001 GENETIC ANALYSIS AND PHENOTYPIC CORRELATION IN DUCTAL PLATE MALFORMATION

Meha Bhuva*, Richard Sandford, William Griffiths. Addenbrookes Hospital, Cambridge, UK

10.1136/gutjnl-2021-BASL.1

Background and Aims Ductal plate malformation (DPM) includes polycystic liver disease (PCLD), multiple biliary hamartomas $(\mathrm{MBH})$, congenital hepatic fibrosis $(\mathrm{CHF})$ and Caroli disease. We sought to identify the genetic basis of a cohort of hitherto undefined DPM.

Methods 34 unrelated adults with presumed congenital liver disease underwent extended genetic analysis via a clinical exome panel (PKD1, PKD2, PKHD1, SEC63, PRKCSH, GANAB, ALG8, DNAJB11, LRP5). Clinical details and phenotypic correlation were analysed.

Results Of the 34 patients screened, genetic variants were identified in 20:

Heterozygous variants in GANAB $(n=4: 3$ females, 1 male, mean age 56) were associated with a variety of mild PCLD phenotypes (with and without renal cysts, with Caroli's and with $\mathrm{MBH})$. Variants were pathogenic in 2 cases and were variants of unknown significance (VUS) in 2 cases (both deleterious).

Heterozygous variants in PRKCSH $(n=2$ : both female, mean age 62) were associated with PCLD without renal cysts including 1 VUS (deleterious) and 1 novel pathogenic variant in a patient considered for liver transplantation.

Heterozygous variants in the SEC63 $(n=4: 3$ females, 1 male, mean age 60) were associated with largely asymptomatic PCLD mostly without renal cysts including 3 pathogenic (all novel) and 1 VUS (deleterious).

PKD1 $(n=3)$ and PKD2 $(n=2)$ heterozygous gene variants (4 pathogenic including 2 novel) were associated with polycystic kidney and liver disease. All 5 were female (mean age 48) and with significant family history.

Variants in PKHD1 $(n=5: 4$ male, 1 female, mean age 67 years) were mostly compound heterozygous and had a variety of phenotypes including $\mathrm{CHF}(\mathrm{n}=2$, both liver transplanted), $\mathrm{CHF} / \mathrm{MBH}(\mathrm{n}=1$, portal hypertension), $\mathrm{MBH} /$ Caroli $(\mathrm{n}=1$, kidney transplanted) and PCLD without renal cysts $(n=1)$.

In 14/34 patients, no genetic variants were identified. These included $3 \mathrm{CHF}, 3 \mathrm{MBH}$ and 8 PCLD patients.

Conclusion Patients with DPM display a spectrum of disease phenotypes ranging in severity from asymptomatic, incidental diagnosis to end-stage liver disease. We identified the genetic cause in nearly $60 \%$ of a previously undefined cohort of DPM patients and discovered several novel variants. Of note, a significant proportion had meaningful variants in GANAB, PRKCSH and SEC63. PRKCSH and SEC63 variants were associated with isolated PCLD. GANAB and PKHD1 mutations were associated with mixed phenotypes, the latter found in mostly males and with a strong propensity for CHF. GANAB and SEC63 phenotypes were mild. Our findings support the use of bespoke gene panels in suspected DPM.

\section{DEEP SEQUENCING OF HCC ENDOTHELIUM REVEALS AN ACTIVE ROLE IN IMMUNOSUPPRESSION AND HIGHLIGHTS THE ECTO NUCLEOTIDASE CD73 AS A POTENTIAL THERAPEUTIC TARGET}

\footnotetext{
${ }^{1,2}$ Rosemary Faulkes* ${ }^{*}{ }^{1,2}$ Joanne O'Rourke, ${ }^{2}$ Owen Cain, ${ }^{1}$ Daniel Patten, ${ }^{1}$ Alex Wilkinson, ${ }^{2}$ Tahir Shah, ' ${ }^{1}$ Christopher Weston, ${ }^{1,2}$ Shishir Shetty. 'University of Birmingham, Birmingham, UK; ${ }^{2}$ University Hospitals Birmingham NHS Trust, Birmingham, UK
}

10.1136/gutjnl-2021-BASL.2
Introduction Overcoming the immunosuppressive microenvironment in HCC is a major challenge. Better understanding of the cell specific contribution is required to help boost current immunotherapy. Endothelial cells are the gatekeeper for immune cell recruitment and we undertook RNA-sequencing (RNA-seq) of purified human HCC endothelium to help define its contribution to the tumour microenvironment of HCC.

Methods Endothelial cells were isolated from liver tissue using a validated method of Ulex-lectin binding. RNAseq was performed on endothelium from primary liver tumours and matched non-tumour endothelial cells. To explore the upregulation of CD73 from the sequencing data we undertook immunohistochemistry for CD73 in a cohort of human HCC. Immunofluorescence for CD73 was performed on isolated liver sinusoidal endothelial cells (LSEC).

Results Analysis of paired tumour and distal non-tumour samples taken from five patients who underwent surgical resection was performed. 45 genes were identified as being significantly differentially expressed between the tumour and non-tumour endothelium (adjusted $\mathrm{p}$ value <0.05). 41 genes were upregulated in the tumour endothelium and 4 downregulated. Pathway analysis revealed 83 pathways that were down regulated (adjusted $\mathrm{p}$ value $<0.05$ ) and these were further grouped into seven key clusters. These clusters were all related to immune related pathways: leucocyte mediated immunity; leucocyte mediated toxicity; leucocyte proliferation; cell killing; exocytosis; cytokine. We focused on CD73 which has a well-established immunosuppressive function. Immunohistochemistry for CD73 on 100 HCC sections confirmed that the protein is present on vascular endothelium and in HCC tumours. The pattern of expression was different in tumour compared to matched non-tumour control, with a peri-membranous staining pattern and variable sinusoidal staining. We also confirmed cell membrane and intracellular expression of CD73 in cultured primary human LSEC using dual colour immunofluorescence.

Conclusion Transcriptomic analysis of human HCC endothelium demonstrates a strong immunosuppressive signature. Interestingly the majority of differentially expressed genes were upregulated, suggesting that the endothelium plays an active role in immunosuppression and directly targeting these pathways could boost the efficacy of other immunotherapies. Validating these findings, CD73 expression was increased in cancer specimens. Furthermore, CD73 is expressed in isolated liver endothelium and, given its functional role in immunosuppression, endothelial CD73 may contribute to the immune HCC microenvironment and could be a promising target for immunotherapy.

\section{A NOVEL NURSE-LED EARLY POST-DISCHARGE CLINIC IS ASSOCIATED WITH FEWER READMISSIONS AND LOWER MORTALITY FOLLOWING AN INDEX HOSPITALISATION WITH DECOMPENSATED CIRRHOSIS}

Benjamin Giles*, Kirsty Fancey, Karen Gamble, Mohid Malik, Zeshan Riaz, Joanna Dowman, Andrew Fowell, Richard Aspinall. Portsmouth Hospitals University NHS Trust, Portsmouth, UK

\subsection{6/gutjnl-2021-BASL.3}

Background Patients hospitalised with decompensated cirrhosis (jaundice, variceal bleeding, ascites or hepatic encephalopathy) have high rates of early, unplanned readmission, associated 